\title{
Detección de anticuerpos y factores de riesgo asociados con Toxoplasma gondii en animales silvestres en un parque zoológico
}

\author{
Detection of antibodies and risk factors associated with Toxoplasma gondii in wild \\ animals in a zoo
}

\author{
Rosa Pinedo V. ${ }^{1,5}$, Amanda Chávez V. ${ }^{1}$, Karina Muñoz D. ${ }^{2}$, Omar Gonzáles-Viera ${ }^{3}$, \\ Eva Casas A. ${ }^{1}$, Deisy Abad-Ameri ${ }^{1}$, Eglinton Villacaqui ${ }^{4}$
}

\section{Resumen}

El objetivo del estudio fue determinar la presencia de anticuerpos contra Toxoplasma. gondii en animales silvestres de los órdenes Carnivora, Primates, Perissodactyla, Cetartiodactyla y Rodentia en el zoológico del Patronato del Parque de las Leyendas, Lima, Perú, así como identificar los factores de riesgo que intervienen en su presentación, estableciendo el rol que cumplen felinos domésticos (Felis silvestris f. catus) y roedores del género Rattus errantes y libres en el establecimiento en estudio en la transmisión del parásito. Se tomaron muestras de sangre de 332 animales (Carnivora: 75, Primates: 71, Perissodactyla: 32, Cetartiodactyla: 134, Rodentia: 20); así como a 41 felinos domésticos y 124 roedores del género Rattus. Además, muestras de cerebro, corazón, hígado y diafragma de los roedores Rattus spp para el análisis histopatológico e inmunohistoquímico. Las muestras de suero fueron analizadas mediante hemaglutinación indirecta (1:64 - 1:2048), considerando como positivo a títulos $\geq 1 / 64$. Se determinó anticuerpos (IgG, IgM) mediante 2-Mercaptoetanol para determinar infecciones agudas y crónicas. En la evaluación de los factores de riesgo se consideraron las variables orden, sexo, tiempo en la institución $(<5 ; 6-10 ;>10$ años) y tipo de recinto (abierto y cerrado), evaluándose mediante análisis de regresión logística múltiple ajustado a Stepwise. La frecuencia de anticuerpos anti-T. gondii en animales silvestres en cautiverio fue de 77.1

${ }^{1}$ Laboratorio de Microbiología y Parasitología Veterinaria, Facultad de Medicina Veterinaria, Universidad Nacional Mayor de San Marcos, Lima, Perú

${ }^{2}$ Patronato del Parque de las Leyendas (PATPAL), Lima, Perú

${ }^{3}$ Facultad de Medicina Veterinaria, Universidad de California, Davis, Estados Unidos

${ }^{4}$ Laboratorio de Epidemiología Veterinaria y Salud Pública, Facultad de Medicina Veterinaria y Zootecnia, Universidad Cientifica del Sur, Lima, Perú

${ }^{5}$ E-mail: rosita_vet99@gmail.com

Recibido: 22 de junio de 2018

Aceptado para publicación: 11 de febrero de 2019 
$\pm 4.4 \%$, con reactividad serológica principal de $\operatorname{IgG}(73.4 \%$, casos crónicos) y fuerte respuesta serológica $(1 / 2048)$ en el $53.9 \%$ de los animales. Los factores de riesgo asociados a la transmisión de $T$. gondii fueron el orden, animales con más de 10 años en la institución (OR: 4.18) y en recinto abierto $(3.99)(\mathrm{p}<0.05)$. Los roedores y gatos que deambulan en el zoo exhibieron una baja $(11.3 \pm 6.0)$ y moderada $(58.5 \pm 15.2)$ frecuencia de anticuerpos anti-T. gondii, respectivamente. Se detectaron quistes tisulares en musculatura cardiaca y esquelética (diafragma) en los roedores ( $4 \%, 5 / 124)$, pero no se pudo demostrar la presencia de $T$. gondii mediante inmunohistoquímica.

Palabras clave: Toxoplasma gondii; animales silvestres; Rattus sp; Felis silvestris $\mathrm{f}$. catus; anticuerpos

The aim of this study was to determine the presence of antibodies against Toxoplasma. gondii in wild animals of the orders Carnivora, Primates, Perissodactyla, Cetartiodactyla and Rodentia in the zoo of the Patronato del Parque de las Leyendas, Lima, Peru, as well as to identify the risk factors associated to toxoplasmosis, establishing the role in the transmission of the parasite of domestic felines (Felis silvestris f. catus) and rodents of the genus Rattus running free in the zoo. Blood samples were taken from 332 animals (Carnivora: 75, Primates: 71, Perissodactyla: 32, Cetartiodactyla: 134, Rodentia: 20); as well as in 41 domestic felines and 124 rodents of the genus Rattus. In addition, samples of brain, heart, liver and diaphragm from the rodents for histopathological and immunohistochemical analysis. Serum samples were analysed by indirect hemagglutination (1:64 -1:2048), considering as positive titres greater $\geq 1 / 64$. Antibodies (IgG, IgM) were determined by 2-Mercaptoethanol to determine acute and chronic infections. In the evaluation of the risk factors, the variables order, sex, time in the institution $(<5,6-10,>10$ years) and type of enclosure (open and closed) were analysed by multiple logistic regression analysis adjusted to Stepwise. The frequency of anti-T. gondii antibodies in wild animals in captivity was $77.1 \pm 4.4 \%$, with serological main reactivity of IgG (73.4\%, chronic cases) and strong serological response (1/2048) in $53.9 \%$ of the animals. The risk factors associated with the transmission of $T$. gondii were the order, animals with more than 10 years in the zoo (OR: 4.18) and exhibited in open area (3.99) $(\mathrm{p}<0.05)$. Rodents and cats that roam in the zoo had a low $(11.3 \pm 6.0)$ and moderate $(58.5$ $\pm 15.2)$ frequency of anti-T. gondii antibodies respectively. Tissue cysts were detected in cardiac and skeletal muscles (diaphragm) in rodents (4\%,5/124), but the presence of $T$. gondii could not be demonstrated by immunohistochemistry.

Key words: Toxoplasma gondii; wild animals; Rattus sp; Felis silvestris f. catus; antibodies

\section{INTRODUCCIÓN}

Los parques zoológicos son espacios de concentración de animales silvestres que cumplen un rol en la conservación de la biodiver- sidad, además de ser centros de investigación y de educación para la población. La participación de la fauna silvestre como reservorios o portadores de zoonosis es de gran importancia, pues constituyen componentes vitales en el ciclo epidemiológico de 
enfermedades que afectan a los seres humanos y animales domésticos (Acha y Szyfres 2003; Pavlin et al., 2009, Cutler et al., 2010). Algunos estudios han estimado que el $60 \%$ de los patógenos emergentes que afectan al hombre proceden de los animales y que más del $70 \%$ de estos tienen origen en la fauna silvestre (Cutler et al., 2010).

Toxoplasma gondii se encuentra presente en todas las latitudes, tanto en poblaciones humanas como en más de 300 especies de mamíferos domésticos y silvestres y en cerca de 30 especies de aves de corral y silvestres. Es un agente patógeno de gran importancia médica y veterinaria, con consecuencias diversas y de intensidad variable en función de la especie, edad y estado inmunitario (Dubey y Jines, 2008; Dubey, 2010).

Los felinos (domésticos y silvestres) son los hospederos definitivos de T. gondii, eliminando ooquistes no esporulados al medio ambiente a través de las heces, los cuales luego de esporular constituyen la forma infectante para otras especies animales, así como para el mismo felino (Cordero del Campillo et al., 1999). La infección puede transmitirse, además, por quistes tisulares presentes en la carne de ratones, aves y herbívoros (Dubey, 2010).

En el Perú se han realizado varios estudios sobre prevalencia de anticuerpos contra T. gondii en ovinos (44\%), cerdos (33.6\%), alpacas $(44.5 \%)$, caninos $(24 \%)$ y felinos (11\%) (Gómez et al., 2003; Ruiz et al., 2012; Cerro et al., 2014; Bernal et al., 2015; Luyo et al., 2017). Sin embargo, los estudios en animales silvestres son escasos; entre ellos, el trabajo realizado por Navarro et al. (2015) en carnívoros y el de Muñoz et al. (2005), quiénes reportaron una alta prevalencia $\mathbf{( 9 0 . 3 \% )}$ en primates en cautiverio de la especie Cebus apella.
Se han reportado frecuencias variables de $T$. gondii en zoológicos de varios países. En la Republica Checa, España y EEUU se han determinado seroprevalencias en carnívoros $(47-90 \%)$, primates $(22-46 \%)$, perisodáctilos (33\%) y artiodáctilos (22-25\%) (Gorman et al., 1986; Sedlák y Bártová, 2006; Sobrino et al., 2007). Las frecuencias altas en carnívoros se deben probablemente a los hábitos de alimentación por consumo de carne infectada, habiéndose observado prevalencias de hasta $100 \%$, aunque clínicamente inaparentes en animales de las familias Hyaenidae, Mustelidae, Ursidae y Viverridae del orden Carnivora (Sobrino et al., 2007). Sin embargo, herbívoros como canguros, lémures y koalas, así como primates neotropicales suelen estar relacionados a mortandad aguda de curso fulminante (Acha y Szyfres, 2003; Epiphanio et al., 2003; De Camps et al., 2008).

Diversos factores epidemiológicos juegan un papel importante en la transmisión de $T$. gondii en los animales silvestres en cautiverio. La infección puede ser mantenida por la presencia de roedores, palomas y felinos (Dubey, 2010). Por otro lado, diversos vectores (cucarachas, moscas coprófilas, gusanos de tierra, etc.) pueden actuar como hospederos de transporte de ooquistes fecales que son consumidos por algunas especies de animales silvestres, realizando una diseminación mecánica (Acha y Szyfres, 2003).

Por lo antes señalado, el objetivo del presente estudio fue determinar la presencia de anticuerpos contra Toxoplasma gondii en animales silvestres en cautiverio de los órdenes Carnivora, Primates, Perissodactyla, Cetartiodactyla y Rodentia, así como identificar los factores de riesgo que intervienen en su presentación, estableciendo el rol que cumplen los felinos domésticos (Felis silvestris f. catus) y roedores del género Rattus que deambulan en estado libre en el zoológico, en la transmisión del parásito. 


\section{Materiales y Métodos}

\section{Lugar del Estudio}

El estudio se realizó en el Zoológico del Patronato del Parque de las Leyendas, ubicado en el distrito de San Miguel, Lima, a 50 msnm, entre enero de 2014 y diciembre de 2015. El procesamiento de las muestras se llevó a cabo en el Laboratorio de Parasitología de la Facultad de Medicina Veterinaria (FMV) de la Universidad Nacional Mayor de San Marcos (UNMSM), Lima, Perú. Los animales en estudio fueron tratados bajo normas y principios de guías internacionales basadas en principios para la investigación biomédica que involucra animales (Bankowski y Howard-Jones, 1986).

\section{Animales Silvestres y Muestras}

Se colectaron muestras de 332 animales distribuidas en 51 especies silvestres (Cuadros 1 y 2), que formaron parte del control sanitario rutinario del parque zoológico. Las especies pertenecían a cinco órdenes: Carnivora $(n=75)$, Primates $(n=71)$, Perissodactyla ( $n=32)$, Cetartiodactyla $(\mathrm{n}=134)$ y Rodentia $(\mathrm{n}=20)$. La contención de los animales se hizo con redes y/o sedación (xilacina y ketamina). Muestras de sangre fueron obtenidas a través de la punción de la vena braquial, yugular o femoral, usando vacutainers estériles. Las muestras fueron identificadas y trasladadas con refrigerantes al laboratorio para su procesamiento y análisis.

\section{Felinos Domésticos y Muestras}

Se obtuvieron muestras sanguíneas de 41 felinos domésticos (Felis silvestris $\mathrm{f}$. catus), que deambulaban en forma libre en el parque zoológico. Esos animales fueron capturados mediante el uso de trampas caseras tipo Tomahawk y fueron sometidos a control reproductivo (castración, ovariohisterectomía), como parte del manejo sanitario del centro. Las muestras de sangre fueron obtenidas mediante punción de la vena cefálica, previa anestesia con xilacina $(1 \mathrm{mg} / \mathrm{kg}) \mathrm{y}$ ketamina $(10 \mathrm{mg} / \mathrm{kg})$.

\section{Roedores y Muestras}

Los roedores $(\mathrm{n}=124)$ fueron atrapados durante un periodo de 12 meses (marzo 2014 a marzo 2015), mediante el uso de trampas Tomahawk de captura viva $(8 \times 9 \times 23 \mathrm{~cm})$. Se colocaron 30 trampas en lugares estratégicos y debidamente identificadas mediante la utilización de un GPS, a distancia no mayor de cinco metros en forma radial y revisadas al día siguiente de su colocación. Los roedores fueron anestesiados mediante la inhalación de cloroformo e inyección de ketamina $(100 \mathrm{mg} / \mathrm{kg})$. La muestra de sangre se obtuvo mediante punción intracardiaca y los animales fueron sacrificados mediante sobredosis de pentobarbital sódico.

Se registraron parámetros morfo-métricos para establecer la especie de roedor (longitud total, largo de cola, patas y orejas) además del sexo y peso. Para la edad, pesos mayores de 130 y 200 g en Rattus rattus y Rattus norvergicus son considerados como ejemplares adultos, respectivamente (Kataranovski et al., 1994; Milazzo et al., 2010). En la manipulación de los roedores se siguieron los estándares de bioseguridad y normas de procesamiento, según los protocolos del Centro de Enfermedades Infecciosas y Prevención de Atlanta (Mills et al., 1998).

\section{Anticuerpos Anti-T. gondii}

Los anticuerpos contra $T$. gondii se determinaron mediante la prueba de hemaglutinación indirecta (HAI) con un «kit» comercial, siguiendo el protocolo del fabricante (Wiener Lab, 2008), Se hicieron diluciones de la muestra de 1:64 a 1:2048 en felinos y animales silvestres y hasta 1:4096 en roedores (Rattus spp), considerándose como positivos los títulos iguales o mayores a $1 / 64$. Reacciones positivas fueron consideradas en aquellos sueros que mostraron aglu- 
Cuadro 1. Especies silvestres contempladas en el estudio (Parte I)

\begin{tabular}{|c|c|c|c|}
\hline Orden / Familia & Especie & Nombre común & N. ${ }^{\circ}$ \\
\hline Carnivora & & & 75 \\
\hline \multirow[t]{3}{*}{ Ursidae } & Ursus americanus & Oso negro & 2 \\
\hline & Ursus arctos arctos & Oso pardo & 2 \\
\hline & Tremarctos ornatus & Oso de anteojos & 7 \\
\hline \multirow[t]{8}{*}{ Felidae } & Panthera leo & León & 10 \\
\hline & Leopardus colocolo & Gato del Pajonal & 7 \\
\hline & Leopardus tigrinus & Oncilla & 6 \\
\hline & Panthera onca & Otorongo & 9 \\
\hline & Panthera tigris & Tigre & 3 \\
\hline & Leopardus pardalis & Tigrillo & 2 \\
\hline & Puma concolor & Puma & 3 \\
\hline & Leopardus wiedii & Margay & 1 \\
\hline \multirow[t]{2}{*}{ Mustelidae } & Eira barbara & Tayra & 3 \\
\hline & Pteronura brasiliensis & Lobo de río / nutria gigante & 1 \\
\hline \multirow[t]{3}{*}{ Canidae } & Lycalopex culpaeus & Zorro andino & 3 \\
\hline & Lycalopex griseus & Zorro plateado & 1 \\
\hline & Canis lupus familiaris & Perro Peruano sin Pelo & 6 \\
\hline \multirow[t]{2}{*}{ Procyonidae } & Nasua nasua & Coatí & 4 \\
\hline & Potos flavus & Chosna & 5 \\
\hline \multirow{3}{*}{$\begin{array}{l}\text { Primates } \\
\text { Cebidae }\end{array}$} & & & 71 \\
\hline & Cebus apella & Mono machín negro & 30 \\
\hline & Cebus albifrons & Mono machín blanco & 5 \\
\hline Aotidae & Aotus nancymaae & $\begin{array}{l}\text { Mono nocturno o } \\
\text { musmuqui }\end{array}$ & 7 \\
\hline Pitheciidae & Pithecia monachus & Mono huapo negro & 8 \\
\hline \multirow[t]{3}{*}{ Atelidae } & Lagothrix lagotricha & Mono choro común & 9 \\
\hline & Ateles chamek & Mono maquisapa negro & 4 \\
\hline & Ateles belzebuth & Mono maquisapa cenizo & 1 \\
\hline \multirow[t]{3}{*}{ Cercopithecidae } & Papio hamadryas & Papión sagrado & 4 \\
\hline & Macaca mulatta & Macaco & 1 \\
\hline & Chlorocebus aethiops & Mono tota o verde & 1 \\
\hline Hominidae & Pan troglodytes & Chimpancé & 1 \\
\hline
\end{tabular}

tinación del antígeno, caracterizada por la formación de una malla o de por lo menos $50 \%$ del área de la cavidad de la microplaca. La formación de botón compacto fue considerada como reacción negativa. En todos los ensayos se utilizaron controles positivos $\mathrm{y}$ negativos. Además, en animales seropositivos se determinó la presencia de anticuerpos (IgG e IgM) mediante el uso del 2-Mercaptoetanol para dilucidar si el tipo de infección es aguda o crónica. 
Cuadro 2. Especies silvestres contempladas en el estudio (Parte II)

\begin{tabular}{|c|c|c|c|}
\hline Orden / Familia & Especie & Nombre común & $\mathrm{N} .^{\circ}$ \\
\hline Cetartiodactyla & & & 134 \\
\hline \multirow[t]{4}{*}{ Bovidae } & Ovis aries & Oveja & 24 \\
\hline & Ovis musimon & Muflón & 1 \\
\hline & Capra hircus & Cabra alpina & 48 \\
\hline & Bubalus arnee & Búfalo de agua & 3 \\
\hline \multirow[t]{2}{*}{ Cervidae } & Hippocamelus antisensis & Taruca & 2 \\
\hline & Odocoileus virginianus & Venado cola blanca & 30 \\
\hline \multirow[t]{5}{*}{ Camelidae } & Vicugna vicugna & Vicuña & 6 \\
\hline & Lama glama & Llama & 3 \\
\hline & pacovicuña & Pacovicuña & 1 \\
\hline & Vicugna pacos & Alpaca & 7 \\
\hline & Lama guanicoe & Guanaco & 4 \\
\hline Tayassuidae & Pecari tajacu & Sajino & 4 \\
\hline Giraffidae & Giraffa camelopardalis & Jirafa & 1 \\
\hline \multicolumn{3}{|l|}{ Perissodactyla } & 32 \\
\hline Tapiridae & Tapirus terrestris & Tapir amazónico & 15 \\
\hline \multirow[t]{3}{*}{ Equidae } & Equus caballus & Caballo & 10 \\
\hline & Equus grevyi & Cebra de Grevy & 4 \\
\hline & Equus quagga & Cebra de Grant & 3 \\
\hline Rodentia & & & 20 \\
\hline \multirow[t]{2}{*}{ Dasyproctidae } & Dasyprocta punctata & Añuje dorado & 3 \\
\hline & Dasyprocta fuliginosa & Añuje común & 4 \\
\hline \multirow[t]{2}{*}{ Caviidae } & Hydrochoerus hydrochaeris & Ronsoco & 2 \\
\hline & Dolichotis patagonum & Mara & 9 \\
\hline Hystricidae & Hystrix cristata & Puerco espín africano & 2 \\
\hline
\end{tabular}

\section{Análisis Histológico e Inmunohistoquí- mico en Rattus spp}

Muestras de tejidos (cerebro, corazón, hígado y diafragma) de los roedores fueron fijadas en formalina tamponada al $10 \% \mathrm{du}-$ rante 48 horas y posteriormente procesadas mediante métodos rutinarios para microscopía óptica para su tinción con HematoxilinaEosina (HE). Se realizaron cortes, que se colocaron en laminillas cubiertas con poli-Llisina. Se utilizó la técnica de anticuerpo pri- mario y polímero conjugado. Como anticuerpo primario se utilizó un anticuerpo comercial: antisuero hiperinmune caprino anti- $T$. gondii, a una dilución de 1:300 y un polímero conjugado como anticuerpo secundario de conejo anti-caprino IgG. Para revelar la reacción inmunológica se utilizó el reactivo comercial 3,3'-diaminobezidina tetrahydrochloride (DAB) para identificar estructuras con inmunopositividad a $T$. gondii (Lacave y Caballero, 2012). 


\section{Encuesta Epidemiológica}

Por cada animal silvestre se aplicó una encuesta epidemiológica con información concerniente a la orden, especie, edad, sexo, alimentación, recinto de exhibición y tiempo en la institución. Asimismo, se obtuvo información concerniente a cada roedor (parámetros morfométricos, edad estimada, sexo y peso) y felino doméstico (edad, sexo).

\section{Análisis de la Información}

El análisis de los factores de riesgo en animales silvestres fue efectuado en dos etapas utilizando el análisis de regresión logística múltiple con el modelo de ajuste de Stepwise. En la primera, cada variable se cruzó con la variable dependiente (seroreactor positivo a $T$. gondii) donde se obtuvieron valores de Odds ratio (OR) crudos para cada variable de exposición. Aquellas que presentaron un valor de $\mathrm{p}<0.05$ por el test de Chicuadrado fueron incluidas en el análisis multivariado, utilizando la regresión logística múltiple (Hosmer y Lemeshow, 2000), para la definición de un modelo que mejor identificara los factores de riesgo, con un nivel de significancia de 5\%. El ajuste del modelo final fue verificado con la prueba de Stepwise. Los análisis fueron realizados con el programa STATA v. 14. La asociación entre las variables epidemiológicas (orden [ $\mathrm{n}=5]$, sexo, alimentación [herbívoro, omnívoro, carnívoro], tiempo en la institución [0-5, 6-10,>10] y recinto de exhibición [abierto, cerrado]) y seropositividad para $T$. gondii fue efectuada con el cálculo OR e intervalo de confianza al 95\% (IC 95\%).

La frecuencia de $T$. gondii en roedores y gatos errantes fue expresada en porcentaje con su respectivo intervalo de confianza. La posible asociación entre las variables con el parasitismo fue analizada mediante la prueba de Chi cuadrado. Se utilizó estadística descriptiva para el estudio histopatológico en muestras de tejidos de roedores.

\section{Consideraciones Éticas}

Todos los procedimientos del presente estudio contemplaron los lineamientos de las buenas prácticas clínicas y de ética en investigación biomédica y con las normas reglamentadas por el Comité de Ética para el Uso de Animales de Experimentación de la FMVUNMSM (Expediente 2014-02).

\section{Resultados}

La seroprevalencia de ocurrencia natural de infección por $T$. gondii en animales silvestres criados en cautiverio fue de $77.1 \pm$ $4.4 \%$, siendo mayor de $70 \%$ en animales de las órdenes Carnivora, Primates, Cetartiodactyla y Rodentia y de 56\% en animales del orden Perissodactyla, resultado que denota una amplia distribución del agente infeccioso (Cuadro 3). Asimismo, el 53.9\% (138/224) de los animales presentaron serología positiva asociada a títulos serológicos elevados $(1 / 2048)$ en todas los órdenes en estudio, a excepción de los Peryssodactyla, cuyo máximo título fue de 1/512. Además, el 73.4\% (188/256) de animales seroreactores a $T$. gondii mostraron principalmente anticuerpos IgG, compatibles con casos crónicos y solo el $26.6 \%(68 / 256)$ fueron compatibles con casos agudos (IgM), como resultado del examen de HAI-mercaptoetanol (Cuadro 3).

El análisis de regresión logística sin ajuste indicó que la variable sexo y alimentación no constituyeron factores de riesgo para la presentación de la parasitosis; sin embargo, hubo asociación estadística significativa $(p<0.05)$ con las variables orden, tiempo del animal en la institución y recinto de exhibición (Cuadro 4). El análisis de regresión logística múltiple ajustado a Stepwise (Cuadro 5) mostró que las variables independientes mantuvieron significancia $(\mathrm{p}<0.05)$ estadística con algunos cambios en la magnitud de la asociación de los riesgos ajustados respecto 
Cuadro 3. Comportamiento serológico anti- $T$. gondii en animales silvestres en cautiverio (Zoológico del Patronato del Parque de las Leyendas), Lima, Perú (2014-2015)

\begin{tabular}{|c|c|c|c|c|c|c|c|c|c|c|}
\hline \multirow[b]{2}{*}{ Orden } & \multirow[b]{2}{*}{ Familia $^{1}$} & \multicolumn{6}{|c|}{ Titulación } & \multirow[b]{2}{*}{$\begin{array}{c}\text { Seropositivos } \\
\quad \text { / Total }\end{array}$} & \multicolumn{2}{|c|}{ Anticuerpo } \\
\hline & & & $\stackrel{\infty}{\stackrel{\infty}{\dddot{I}}}$ & $\begin{array}{l}\stackrel{\circ}{\longrightarrow} \\
\stackrel{\sim}{*}\end{array}$ & $\stackrel{\sim}{n}$ & $\begin{array}{l}\stackrel{ \pm}{0} \\
\stackrel{0}{-}\end{array}$ & $\stackrel{\substack{+\stackrel{\leftrightarrow}{+}}}{=}$ & & $\operatorname{IgM}$ & IgG \\
\hline \multirow{6}{*}{$\begin{array}{l}\text { Carnivora } \\
(76.0 \%)\end{array}$} & & 6 & 7 & 6 & 10 & 4 & 24 & $57 / 75$ & 38.0 & 66.7 \\
\hline & Ursidae & 0 & 1 & 1 & 1 & 0 & 7 & $10 / 11$ & 30 & 70 \\
\hline & Felidae & 3 & 4 & 5 & 6 & 3 & 14 & $35 / 41$ & 34.3 & 65.7 \\
\hline & Mustelidae & 3 & 1 & 0 & 0 & 0 & 0 & $4 / 4$ & 0 & 100 \\
\hline & Canidae & 0 & 0 & 0 & 0 & 1 & 1 & $2 / 10$ & 0 & 100 \\
\hline & Procyonidae & 0 & 1 & 0 & 3 & 0 & 2 & $6 / 9$ & 66.6 & 33.3 \\
\hline \multirow{7}{*}{$\begin{array}{l}\text { Primates } \\
(76.7 \%)\end{array}$} & & 2 & 5 & 6 & 5 & 5 & 30 & $53 / 71$ & 30.2 & 60.8 \\
\hline & Cebidae & 0 & 3 & 4 & 2 & 2 & 21 & $32 / 35$ & 9.4 & 90.6 \\
\hline & Aotidae & 1 & 1 & 1 & 0 & 0 & 1 & $4 / 7$ & 75 & 25 \\
\hline & Pitheciidae & 1 & 0 & 0 & 1 & 2 & 2 & $6 / 17$ & 66.7 & 33.3 \\
\hline & Atelidae & 0 & 0 & 0 & 0 & 0 & 5 & $5 / 5$ & 20 & 80 \\
\hline & Cercopithecidae & 0 & 1 & 1 & 1 & 1 & 1 & $5 / 6$ & 80 & 20 \\
\hline & Hominidae & 0 & 0 & 0 & 1 & 0 & 0 & $1 / 1$ & 100 & 0 \\
\hline \multirow{6}{*}{$\begin{array}{l}\text { Cetartiodactyla } \\
(82.1 \%)\end{array}$} & & 6 & 3 & 8 & 11 & 8 & 74 & $110 / 134$ & 23.6 & 76.4 \\
\hline & Bovidae & 3 & 3 & 3 & 5 & 7 & 41 & $62 / 76$ & 22.6 & 77.4 \\
\hline & Cervidae & 2 & 0 & 4 & 4 & 0 & 19 & $29 / 32$ & 17.2 & 82.8 \\
\hline & Camelidae & 0 & 0 & 0 & 2 & 1 & 13 & $16 / 21$ & 25 & 75 \\
\hline & Tayassuidae & 1 & 0 & 1 & 0 & 0 & 0 & $2 / 4$ & 100 & 0 \\
\hline & Giraffidae & 0 & 0 & 0 & 0 & 0 & 1 & $1 / 1$ & 100 & 0 \\
\hline \multirow{3}{*}{$\begin{array}{l}\text { Perissodactyla } \\
(56.2 \%)\end{array}$} & & 5 & 6 & 2 & 5 & 0 & 0 & $18 / 32$ & 5.6 & 94.4 \\
\hline & Tapiridae & 1 & 1 & 0 & 4 & 0 & 0 & $6 / 15$ & 16.7 & 83.3 \\
\hline & Equidae & 4 & 5 & 2 & 1 & 0 & 0 & $12 / 17$ & 0 & 100 \\
\hline \multirow{4}{*}{$\begin{array}{l}\text { Rodentia } \\
(90.0 \%)\end{array}$} & & 2 & 1 & 1 & 4 & 0 & 10 & $18 / 20$ & 33.3 & 66.7 \\
\hline & Dasyproctidae & 1 & 0 & 1 & 2 & 0 & 1 & $5 / 7$ & 60 & 40 \\
\hline & Caviidae & 1 & 1 & 0 & 2 & 0 & 7 & $11 / 11$ & 18.2 & 81.8 \\
\hline & Hystricidae & 0 & 0 & 0 & 0 & 0 & 2 & $2 / 2$ & 50 & 50 \\
\hline Total & & 21 & 22 & 23 & 35 & 17 & 138 & $256 / 332$ & 26.6 & 73.4 \\
\hline
\end{tabular}

${ }^{1}$ Ver las especies involucradas en los cuadros 1 y 2

de los riesgos crudos. Los órdenes Rodentia, Cetartiodactyla, Primates y Carnivora presentaron $8.77,4.93,3.60$ y 2.61 veces más posibilidades de presentar anticuerpos contra $T$. gondii, respectivamente, que los animales del orden Perissodactyla. Asimismo, se encontró un incremento de la frecuencia de T. gondii (72.5-88.6\%) conforme se incrementa los años de permanencia en el centro en cautiverio (Cuadro 5), encontrándose que los animales con más de 10 años en la institución mostraron 4.18 veces más riesgo de infección que animales con permanencia de hasta 5 años $(\mathrm{p}<0.05)$. 
Cuadro 4. Factores de riesgo asociados a Toxoplasma gondii en animales silvestres en cautiverio (Zoológico del Patronato del Parque de las Leyendas), Lima, Perú. Cálculo del Odds Ratio crudo (2014-2015)

\begin{tabular}{|c|c|c|c|c|c|c|c|}
\hline \multirow{2}{*}{ Variables } & \multirow{2}{*}{$\mathrm{N}$} & \multirow{2}{*}{$\begin{array}{c}\text { Positivos } \\
=1 / 64\end{array}$} & \multirow{2}{*}{$\begin{array}{c}\text { Frecuencia } \\
\%\end{array}$} & \multirow{2}{*}{$\begin{array}{l}\text { Odds } \\
\text { ratio }\end{array}$} & \multicolumn{2}{|c|}{ IC $95 \%$} & \multirow{2}{*}{$\begin{array}{c}\text { Valor } \\
\mathrm{p}\end{array}$} \\
\hline & & & & & Mínimo & Máximo & \\
\hline \multicolumn{8}{|l|}{ Orden } \\
\hline Perissodactyla & 32 & 18 & 56.25 & 1 & & & \\
\hline Primates & 71 & 53 & $74.65^{*}$ & 10.35 & 1.50 & 71.27 & 0.018 \\
\hline Carnivora & 75 & 57 & 76.00 & 7.96 & 0.81 & 52.70 & 0.077 \\
\hline Cetartiodactyla & 134 & 110 & $82.09^{*}$ & 7.41 & 2.69 & 20.38 & 0.000 \\
\hline Rodentia & 20 & 18 & $90.00^{*}$ & 8.74 & 1.65 & 46.31 & 0.010 \\
\hline \multicolumn{8}{|l|}{ Sexo } \\
\hline Machos & 115 & 82 & 71.30 & 1 & & & \\
\hline Hembras & 217 & 174 & 80.18 & 1.30 & 0.72 & 2.36 & 0.375 \\
\hline \multicolumn{8}{|c|}{ Tiempo en la institución } \\
\hline 0 a 5 años & 182 & 132 & 72.53 & 1 & & & \\
\hline de 6 a 10 & 71 & 54 & 76.06 & 1.57 & 0.94 & 4.72 & 0.068 \\
\hline$>10$ años & 79 & 70 & $88.61 *$ & 4.89 & 2.44 & 16.12 & 0.000 \\
\hline \multicolumn{8}{|l|}{ Alimentación } \\
\hline Herbívoro & 200 & 156 & 78.00 & 1 & & & \\
\hline Omnívoro & 86 & 64 & 74.42 & 0.19 & 0.050 & 1.37 & 0.11 \\
\hline Carnívoro & 46 & 36 & 78.26 & 0.41 & 0.73 & 4.55 & 0.60 \\
\hline \multicolumn{8}{|l|}{ Exhibición } \\
\hline Cerrado & 32 & 18 & 56.25 & 1 & & & \\
\hline Abierto & 300 & 238 & $79.33^{*}$ & 4.62 & 1.29 & 16.54 & 0.019 \\
\hline Total & 332 & 256 & $77.1 \pm 4.4$ & & & & \\
\hline
\end{tabular}

$* p<0.05$

La seropositividad fue significativamente superior $(\mathrm{p}<0.05)$ en animales que eran mantenidos en recintos de exhibición abiertos (79.3\%; Cuadro 4) en comparación aquellos que permanecían en recintos cerrados (56.25), mostrando OR: 3.99 (Cuadro 5).

La frecuencia de anticuerpos anti- $T$. gondii fue de $58.54 \%(24 / 41)$ en los gatos (Felis silvestris f. catus) y de 11.29\% (14/124) en las ratas (Cuadros 6 y 7). Asimismo, la mayoría de los gatos expuestos a $T$. gondii evidenciaron la fase crónica de la infección
(IgG 95.8\%, [23/24]) y el 66\% (16/24) de gatos seropositivos mostraron títulos altos de anticuerpos (1/2048) (Cuadro 6).

No se observaron efectos significativos entre especie, sexo y edad de los roedores (Rattus spp) con la infección por T. gondii (Cuadro 7). Asimismo, el título más frecuente fue $1 / 64(35.7 \%,[5 / 14])$, seguido de $1 / 128$ $(28.57 \%,[4 / 14])$ en los animales serorreactores, evidenciándose similar proporción de anticuerpos $\operatorname{IgM}(57.1 \%$ [8/14]) e $\operatorname{IgG}$ (42.9\% [6/14\%]) (Cuadro 8). 
Cuadro 5. Factores de riesgo asociados a Toxoplasma gondii en animales silvestres en cautiverio, (Zoológico del Patronato del Parque de las Leyendas), Lima, Perú. Cálculo de Odss Ratio ajustado ${ }^{1}$ (2014-2015)

\begin{tabular}{|c|c|c|c|c|}
\hline \multirow{2}{*}{ Variables } & \multirow{2}{*}{ Odds Ratio } & \multicolumn{2}{|c|}{ Intervalo de confidencia $95 \%$} & \multirow{2}{*}{ Valor $\mathrm{p}$} \\
\hline & & Mínimo & Máximo & \\
\hline \multicolumn{5}{|l|}{ Orden } \\
\hline Primates & 3.61 & 1.17 & 11.07 & 0.025 \\
\hline Carnivora & 2.61 & 1.04 & 6.55 & 0.040 \\
\hline Cetartiodactyla & 4.93 & 2.07 & 11.74 & 0.000 \\
\hline Rodentia & 8.77 & 1.69 & 45.44 & 0.010 \\
\hline Perissodactyla & 1 & & & \\
\hline \multicolumn{5}{|l|}{ Tiempo en la institución } \\
\hline$>10$ años & 4.18 & 1.84 & 9.48 & 0.001 \\
\hline$=5$ años & 1 & & & \\
\hline \multicolumn{5}{|l|}{ Exhibición (recinto) } \\
\hline Abierto & 3.99 & 1.37 & 11.6 & 0.011 \\
\hline Cerrado & 1 & & & \\
\hline
\end{tabular}

${ }^{1}$ Regresión logística múltiple ajustado a Stepwise

Cuadro 6. Frecuencia y respuesta serológica anti-Toxoplasma gondii en gatos (Felis silvestris f. catus) errantes libres en un Zoológico de Lima, Perú (2014-2015)

\begin{tabular}{|c|c|c|c|c|c|c|c|c|c|c|}
\hline \multirow[b]{2}{*}{ Sexo } & \multicolumn{6}{|c|}{ Titulación } & \multirow[b]{2}{*}{$\begin{array}{c}\text { Seropositivos } \\
\text { / Total }\end{array}$} & \multirow[b]{2}{*}{$\mathrm{P} \% \pm \mathrm{IC}$} & \multicolumn{2}{|c|}{ Anticuerpo } \\
\hline & $\stackrel{t}{\oplus}$ & $\stackrel{\infty}{\stackrel{\infty}{\leftrightarrows}}$ & 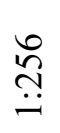 & $\stackrel{\stackrel{\sim}{n}}{\stackrel{n}{-}}$ & $\begin{array}{l}\stackrel{+}{\Delta} \\
\stackrel{\Delta}{-}\end{array}$ & 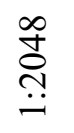 & & & $\operatorname{IgM}$ & $\operatorname{IgG}$ \\
\hline Hembras & 0 & 1 & 1 & 0 & 0 & 5 & $7 / 15$ & 46.67 & 14.3 & 85.7 \\
\hline Machos & 1 & 2 & 1 & 2 & 0 & 11 & $17 / 26$ & 65.38 & 0 & 100 \\
\hline Total & 1 & 3 & 2 & 2 & 0 & 16 & $24 / 41$ & $58.5 \pm 15.2$ & 4.2 & 95.8 \\
\hline
\end{tabular}

En la evaluación histopatológica e inmunohistoquímica se evidenciaron formas parasitarias quísticas (4\%, [5/124]), principalmente en el diafragma, seguido del músculo estriado cardíaco de los roedores. Solo dos animales seroreactores positivos a $T$. gondii con títulos de 1/4096 evidenciaron quistes tisulares $14.28 \%$ (2/14). Sin embargo, no se pudo demostrar la presencia de quistes de $T$. gondii mediante la técnica de inmunohistoquímica en los roedores que evidenciaron quistes tisulares. 
Cuadro 7. Frecuencia anti-Toxoplasma gondii en roedores (Rattus spp) errantes libres en un Zoológico de Lima, Perú (2014-2015)

\begin{tabular}{lccccc}
\hline & $\mathrm{N}^{\circ}$ & Positivos & $\mathrm{P} \% \pm \mathrm{IC}_{95 \%}$ & $\mathrm{X}^{2}$ & Valor de $\mathrm{p}$ \\
\hline Especie & & & & & \\
$\quad$ Rattus rattus & 46 & 6 & 13.04 & - & - \\
$\quad$ Rattus norvegicus & 78 & 8 & 10.26 & 0.22 & 0.636 \\
Sexo & & & & & \\
$\quad$ Hembras & 60 & 4 & 6.67 & - & - \\
$\quad$ Machos & 64 & 10 & 15.63 & 2.48 & 0.115 \\
Edad & & & & & \\
$\quad$ Juvenil-subadulto & 92 & 9 & 9.78 & - & - \\
$\quad$ Adulto & 32 & 5 & 15.63 & 0.81 & 0.368 \\
Total (\% $\%$ IC) & 124 & 14 & $11.29 \pm 6.0$ & & \\
\hline
\end{tabular}

Cuadro 8. Comportamiento serológico anti-T. gondii en roedores (Rattus spp) errantes libres en el Zoológico del Patronato del Parque de las Leyendas, Lima, Perú (2014-2015)

\begin{tabular}{|c|c|c|c|c|c|c|c|c|c|c|c|}
\hline \multirow[b]{2}{*}{ Variable } & & \multicolumn{6}{|c|}{ Titulación } & & \multirow[b]{2}{*}{$\begin{array}{l}\text { Seropositivos } \\
\quad / \text { Total }\end{array}$} & \multicolumn{2}{|c|}{ Anticuerpo } \\
\hline & & بـ & $\stackrel{\infty}{\stackrel{\infty}{\leftrightarrows}}$ & ڤ̊ํㅛ & $\stackrel{\sim}{n}$ & 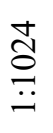 & $\begin{array}{l}\stackrel{\infty}{\stackrel{\oplus}{ஸ}} \\
\stackrel{-}{-}\end{array}$ & $\begin{array}{l}\text { ঃ̊ } \\
\text { \&े } \\
\stackrel{+}{-}\end{array}$ & & $\operatorname{IgM}$ & IgG \\
\hline \multirow[t]{3}{*}{ Especie } & & 6 & 7 & 6 & 10 & 4 & 24 & & $57 / 75$ & 38.0 & 66.7 \\
\hline & Rattus rattus & 3 & 1 & 0 & 0 & 0 & 0 & 2 & $6 / 46$ & 83.3 & 16.7 \\
\hline & R. norvegicus & 2 & 3 & 2 & 1 & 0 & 0 & 0 & $8 / 78$ & 37.5 & 62.5 \\
\hline \multicolumn{12}{|l|}{ Sexo } \\
\hline & Hembras & 1 & 2 & 0 & 1 & 0 & 0 & 0 & $4 / 60$ & 25 & 75 \\
\hline & Machos & 4 & 2 & 2 & 0 & 0 & 0 & 2 & $10 / 64$ & 70 & 30 \\
\hline \multicolumn{12}{|l|}{ Edad } \\
\hline & $\begin{array}{l}\text { Juvenil- } \\
\text { subadulto }\end{array}$ & 4 & 2 & 1 & 1 & 0 & 0 & 1 & 9/92 & 77.8 & 22.2 \\
\hline & Adulto & 1 & 2 & 1 & 0 & 0 & 0 & 1 & $5 / 32$ & 20 & 80 \\
\hline Total & & 5 & 4 & 2 & 1 & 0 & 0 & 2 & $14 / 124$ & 57.1 & 42.9 \\
\hline
\end{tabular}

\section{Discusión}

Los parques zoológicos constituyen un ecosistema artificial cerrado y pueden albergar una amplia diversidad de agentes infecciosos y constituir una fuente de diseminación de patógenos parasitarios para los ani- males silvestres del propio zoológico, animales sinantrópicos, funcionarios y público visitante (Silva et al., 2007).

El presente estudio reportó $77 \%$ de ocurrencia de anticuerpos para la infección por $T$. gondii en mamíferos de los cinco órdenes en estudio, indicando que la infección por este 
patógeno es frecuente en ese establecimiento. La seroprevalencia encontrada es una de las mayores que ha sido reportada en comparación con seroprevalencias entre $24.0 \mathrm{y}$ $53.3 \%$ reportadas en otros parques zoológicos del continente americano (Smitth y Frenkel, 1995; De Camps et al., 2008, Minervino et al., 2010; Pimentel et al., 2009; Alvarado Esquivel et al., 2013) y entre 33.3 y $34.5 \%$ en zoológicos europeos (Sedlák y Bártová, 2006; Alerte, 2008; Tidy et al., 2017).

La comparación entre seroprevalencias de $T$. gondii en parques zoológicos es compleja debido a la variedad de especies silvestres con diversos hábitos de comportamiento, número de especímenes evaluados, técnica de diagnóstico serológico, punto de corte, condiciones climáticas y de higiene a las que se exponen los animales, así como la ubicación geográfica de los parques zoológicos (Sedlák y Bártová 2006; De Camps et al., 2008). En este sentido, Sedlák y Bártová (2006) reportaron en zoológicos de la República Checa y Eslovaquia seroprevalencias de 34.7\% (193/596) en mamíferos silvestres, pudiendo la diferencia ser debida, además, al uso de una técnica de mayor especificidad, como es la técnica de inmunofluorescencia indirecta.

Existe controversia al señalar la dispersión del agente infeccioso en animales silvestres en su habitad natural versus en cautiverio como es el caso de zoológicos. Minervino et al. (2010) señala un mayor riesgo de exposición al agente en animales silvestres en cautiverio en comparación a los de vida libre, dado que los animales en los zoológicos tienen mayores posibilidades de estrés ante las altas densidades de animales y tipos de dieta, además de mayores grados de consanguinidad (Scott, 1988).

El título más frecuente en los animales silvestres seropositivos fue de 1/2048 (53.9\%) y solo el $8.2 \%$ presentó el título más bajo $(1 / 64)$. Información sobre los niveles de anticuerpos es escasa. Damriyasa et al. (2004) proponen que la detección de bajos títulos de anticuerpos en animales puede de- berse a la presencia de reacciones cruzadas con otros parásitos Apicomplexa (Eimeria, Isospora, Sarcocysts), o una disminución de anticuerpos específicos de $T$. gondii posterior a una infección primaria.

La mayoría de los anticuerpos anti- $T$. gondii fueron IgG (73.4\%, [188/332]), cuya presencia indicaría exposición del parásito en algún momento de la vida del animal; desarrollando generalmente una fuerte respuesta inmune, que persistiría toda su vida (Dubey, 2010). Por otro lado, la toxoplasmosis crónica no siempre puede permanecer latente y más aún en animales silvestres en cautiverio, los cuales soportan situaciones de estrés por múltiples motivos. Todo ello llevaría a la reactivación del parásito, conllevando que quistes tisulares conteniendo miles de bradizoitos se interconviertan a taquizoitos y desarrollen parasitemia activa nuevamente (Dubey, 2010).

Se conoce muy poco sobre las manifestaciones clínicas y lesionales ocasionadas por T. gondii en animales silvestres. En el estudio no fue posible observar en forma rutinaria a los animales en busca de signos clínicos relacionados al agente infeccioso; sin embargo, la detección de IgM como marcador de infección aguda (26.6\%, [68/256]), indica que pudieron haberse presentado. Los signos clínicos pueden ser inespecíficos (pirexia, alteraciones digestivas, anorexia, debilidad progresiva, pérdida de peso, letargia, etc.) y pasar desapercibidos o ser fatales en especies susceptibles como canguros (Bermúdez et al., 2009), guepardos (Lloyd y Stidworthy, 2007), gato de Pallas (Basso et $a l ., 2005)$ y monos ardillas (Cedillo-Peláez et al., 2011). Es de mencionar que en el establecimiento se presentó, durante el estudio, un caso de toxoplasmosis activo en Hystrix cristata (puerco espín africano) asociado a signos neurológicos. El diagnóstico fue confirmado por serología (IgM) de HAI y luego de un tratamiento a base de sulfametoxazol + trimetropin $30 \mathrm{mg} / \mathrm{kg}$ i.m. durante cinco semanas presentó la remisión de algunos signos clínicos (ceguera), aunque le quedaron algunas secuelas neurológicas. 
Los valores más altos de frecuencia a $T$. gondii en el presente estudio se registraron en los órdenes Rodentia (90\%), Carnivora $(85 \%)$ y Cetartiodactyla (82\%), seguida del orden Primate $(76 \%)$. Estos resultados difieren de otros reportes que describen una mayor susceptibilidad del orden Carnivora por el consumo de carne, llegándose a reportar hasta frecuencias de 100\% (Sobrino et al., 2007). Es posible que en este caso, animales de los órdenes Rodentia y Cetartyodactila hayan presentado mayores niveles que en el orden Carnivora debido a que la infección es mantenida por la contaminación ambiental, donde los felinos domésticos jugarían un papel importante en la diseminación de ooquistes.

Los Perissodactyla registraron la frecuencia más baja (56\%), lo cual podría ser explicado por el nivel de resistencia a la infección por $T$. gondii en equinos, lo que conlleva a la ausencia de signos clínicos en dichos animales (Al-Khalidi y Dubey, 1979; Silva, 2005; Dubey y Jones 2008; GarcíaBocanegra et al., 2012).

La seroprevalencia de $T$. gondii en animales silvestres en cautiverio se ve influenciada por el tipo de recinto de exhibición de los animales. Aquellos en instalaciones abiertas o al aire libre presentaron 3.99 veces mayor probabilidad de presentar anticuerpos a $T$. gondii que aquellos en recintos cerrados, coincidiendo con el estudio de De Camps et al. (2008) en EEUU. La diferencia encontrada entre ambos tipos de recintos puede deberse a la facilidad con la que se puede producir el contacto con gatos y roedores en el zoológico en las instalaciones al aire libre, aumentando la probabilidad de ingestión de ooquistes y quistes tisulares.

E1 sexo no estuvo asociado a la seroprevalencia de $T$. gondii, tal y como lo reportó Lopes et al. (2011) en el norte de Portugal y Silva et al. (2007) en Brasil. Sin embargo, Miller et al. (2002) señalaron que mamíferos machos en vida libre son más propensos a la infección debido a que recorren largas distancias para establecer y defender su territorio, teniendo, por lo tanto, mayores posibilidades de exposición al agente infeccioso.

La alta frecuencia de anticuerpos de anti- $T$. gondii en gatos errantes o asilvestrados que viven en el parque zoológico (58.5\%) representa una alarmante situación y refuerza la importancia de estos animales en la epidemiología del parásito. Considerando el potencial de $T$. gondii en infecciones experimentales, se puede deducir que un gato asilvestrado en el parque zoológico puede excretar entre 20 a 150 millones de ooquistes durante su vida (Dubey, 1995). No se encontró estudios en este tipo de gatos para su comparación con los hallazgos en este trabajo; sin embargo, Silva et al. (2002) y Troncoso et al. (2015) señalan que gatos de la calle presentan altas seroprevalencias (27$52 \%$ ) debido a un mayor riesgo de exposición por infección a través de ooquistes o de la ingestión de quistes tisulares de pájaros o roedores. Trabajos en gatos de Lima Metropolitana muestran una seroprevalencia inferior (11\%) mediante la técnica de HAI (Cerro et al., 2014).

Se podría postular que los leones, oncillas, otorongos, tigres, tigrillos, pumas y gatos de pajonal también podrían servir como hospederos definitivos y excretores de ooquistes. El papel de felinos silvestres en la transmisión del parásito es incierto a diferencia de lo claramente establecido para felinos domésticos. La capacidad potencial de felinos silvestre para albergar a $T$. gondii ha sido demostrada en felinos en cautiverio en California, EEU, donde 9 de 12 especies tuvieron anticuerpos anti-T. gondii (Riemann et al., 1975). Estudios más recientes en Brasil mostraron tasas de prevalencia entre 52.8 y $66.7 \%$ (Silva et al., 2007; André et al., 2010; Ullmann et al., 2010). Asimismo, se han realizado infecciones experimentales para determinar la excreción de ooquistes a través de las heces en jaguares (Puma yagouaroundi), ocelotes (Felis pardalis), linces (Lynx rufus) y guepardos (Acinonyx 
jubatus) (Mucker et al., 2006). Sin embargo, el papel de estos felinos en la consiguiente contaminación ambiental es menos significativa que la del gato doméstico errante asilvestrado (Weiss y Kim, 2007).

Un factor importante para la infección con $T$. gondii fue el tiempo de exposición en cautiverio, encontrando un OR de 4.18 para el grupo expuesto mayor a 10 años en comparación a los otros. Los animales se encuentran en contacto continuo con el parásito, manteniendo una inmunidad activa y detectable a edades avanzadas (Dubey y Jones, 2008; Richomme et al., 2010).

Los hábitos de alimentación no fueron un factor significativo para la ocurrencia de infección por T. gondii. Tanto herbívoros, carnívoros y omnívoros presentaron similares frecuencias en contraste a otros estudios que muestran mayores prevalencias en carnívoros (Smith y Frenkel, 1995; Dãrãbus et al., 2011). A pesar de que los carnívoros podrían ser más vulnerables debido a una dieta basada en carne fresca producto de la saca por sobrepoblación de ovejas, cabras, búfalos y sajinos del mismo parque zoológico, las cuales albergarían un alto número de quistes tisulares viables. La elevada seroprevalencia en los herbívoros del presente estudio sugiere que estos se exponen principalmente al parásito a través de la ingestión de ooquistes, provenientes de gatos errantes o a través de vectores mecánicos (ropa de los cuidadores, botas, equipo de limpieza, etc.), invertebrados o por roedores, además de aves capaces de moverse libremente en los ambientes (Hill y Dubey, 2002; Spencer et al., 2004).

Los roedores (Rattus spp y Mus musculus) juegan un papel importante en el ciclo de vida de $T$. gondii, y constituyen la fuente principal de infección para gatos domésticos y silvestres. Sin embargo, la frecuencia en Rattus spp (11.29\%) no se correlacionó con la tasa de frecuencia en gatos domésticos errantes (58.54\%) en el parque zoológico. Estos resultados indicarían que las ratas podrían no ser la fuente principal de infec- ción para los gatos, existiendo otro grupo alternativo de infección como ardillas, ratones y aves no evaluados en el presente trabajo. Se debe considerar asimismo, que las investigaciones en roedores (Rattus spp) han sido limitadas, y donde se muestra su baja positividad (Pellizzaro et al., 2017).

Si bien se ha demostrado la persistencia del agente en varias especies silvestres, falta dilucidar la estructura genética de las cepas clonales implicadas en la población expuesta. Existe información sobre el aislamiento de $T$. gondii en fauna silvestre proveniente principalmente de América del Norte, pero también de Brasil (Yai et al., 2009; Pena et al., 2011; Cañón-Franco et al., 2013; Vitalino et al., 2014).

El diagnóstico de la toxoplasmosis puede realizarse por métodos biológicos, serológicos, histológicos y moleculares, cada una con diferente sensibilidad, especificidad y blanco antigénico (Dubey et al., 1995; Lind et al., 1997). En el presente estudio se utilizó la técnica serológica de hemaglutinación indirecta, con un punto de corte $\geq 64$, prueba más accesible en el país, la cual se basa en la propiedad de los anticuerpos anti- $T$. gondii de producir aglutinación en la presencia de eritrocitos sensibilizados con antígenos citoplasmáticos y de membrana del parásito (Wiener Lab, 2008). Sin embargo, se requiere considerar las posibles reacciones cruzadas con otros Apicomplexos (Neospora, Sarcocystis) formadores de quistes tisulares que pertenecen a la misma familia (Sarcocystidae) y, por consiguiente, originar falsos positivos (Nishikawa et al., 2002).

\section{Conclusiones}

- Se detectó una alta frecuencia (77.11 \pm $4.41 \%)$ de anticuerpos contra Toxoplasma gondii en animales silvestres en cautiverio de los órdenes Carnivora, Primates, Cetartiodactyla y Rodentia, con reactividad serológica mayormente 
de IgG (73.4\%, casos crónicos) y fuerte respuesta serológica $(1 / 2048)$ en el $53.9 \%$ de los animales.

- Los factores de riesgo asociados a la transmisión de $T$. gondii para animales de los órdenes Carnivora, Primates, Cetartiodactyla y Rodentia fueron de OR: 2.6, 3.6, 4.9 y 8.7, respectivamente. Asimismo, animales con más de 10 años en el establecimiento (OR: 4.18) y mantenidos en recintos de exhibición abiertos (3.99) $(\mathrm{p}<0.05)$.

- Roedores (Rattus spp) y gatos (Felis silvestris f. catus) errantes libres que deambulan en el centro en cautiverio exhibieron una baja (11.29 \pm 6$)$ y moderada $(58.54 \pm 15.2)$ frecuencia de anticuerpos anti-T. gondii, respectivamente.

- Se detectaron quistes tisulares en musculatura cardiaca y esquelética (diafragma) en el 4\% (5/124) de los roedores evaluados; sin embargo, no se pudo demostrar la presencia de $T$. gondii mediante inmunohistoquímica.

\section{Literatura Citada}

1. Acha PN, Szyfres B. 2003. Zoonosis y enfermedades transmisibles comunes al hombre y animales. $3{ }^{\text {a }}$ ed. Vol III. Washington, EEUU: OPS. 312 p.

2. Alerte VM. 2008. Prévalence de Toxoplasma gondii sur les animaux d'un parc zoologique (Amneville): séroprévalence et isolement du parasite. Tesis Doctoral. Versailles, Francia: Université Paul-Sabatier de Toulouse. $131 \mathrm{p}$.

3. Al-Khalidi NW, Dubey JP. 1979. Prevalence of Toxoplasma gon-dii infection in horses. J Parasitol 65: 331-334.

4. Alvarado-Esquivel C, GayossoDominguez EA, Villena I, Dubey JP. 2013. Seroprevalence of Toxoplasma gondii infection in captive mammals in three Zoos in Mexico City, Mexico. J Zoo Wildlife Med 44: 803-806. doi: 10.1638/ 2013-0032.1
5. André MR, Adania CH, Teixeira RH, Silva KF, Jusi MM, Machado ST, et al. 2010. Antibodies to Toxoplasma gondii and Neospora caninum in captive neotropical and exotic wild canids and felids. J Parasitol 96: 1007-1009. doi: 10.1645/GE-2502.1

6. Bankowski Z, Howard-Jones N. 1986. International guiding principles for biomedical research involving animals (1985). Geneva: WHO. 28 p.

7. Basso W, Edelhofer R, Zenker W, Möstl K, Kübber-Heiss A, Prosl H. 2005. Toxoplasmosis in Pallas' cats (Otocolobus manul) raised in captivity. Parasitology 130: 293-299. doi: 10.1017/ S0031182004006584

8. Bermúdez $R$, Faílde LD, Losada AP, Nieto JM, Quiroga MI. 2009. Toxoplasmosis in Bennett's wallabies (Macropus rufogriseus) in Spain. Vet Parasitol 160: 155-158. doi: 10.1016/ j.vetpar.2008.10.082

9. Bernal D, Suárez, Huanca W, Chávez A. 2015. Prevalencia de toxoplasmosis ovina en dos localidades de Puno, Perú Rev Inv Vet Perú 26: 291-295. doi: 10.15381/rivep.v26i2.11002

10. García-Bocanegra I, Cabezón O, Arenas-Montes A, Carbonero A, Dubey JP, Perea A, Almería S. 2012. Seroprevalence of Toxoplasma gondii in equids from Southern Spain. Parasitol Int 61: 421-424. doi: 10.1016/j.parint.2012.02.003

11. Cañón-Franco WA, Araújo FA, López-Orozco N, Jardim MM, Keid LB, Dalla-Rosa C. 2013. Toxoplasma gondii in free-ranging wild small felids from Brazil: molecular detection and genotypic characterization. Vet Parasitol 197: 462-469. doi: 10.1016/ j.vetpar.2013.07.019

12. Cedillo-Peláez C, Rico-Torres CP, Salas-Garrido CG, Correa D. 2011. Acute toxoplasmosis in squirrel monkeys (Saimiri sciureus) in Mexico. Vet Parasitol 180: 368-371. doi: 10.1016/ j.vetpar.2011.03.012 
13. Cerro L, Rubio A, Pinedo R, Mendesde-Almeida F, Brener B, Labarthe $N$. 2014. Seroprevalence of Toxoplasma gondii in cats (Felis catus, Linnaeus 1758) living in Lima, Peru. Rev Bras Parasitol V 23: 90-93. doi: 10.1590/ S1984-29612014013

14. Cordero del Campillo M, Rojo FD, Martínez AR, Sánchez MC, Hernández S, Navarrete I, et al. 1999. Parasitología veterinaria. Madrid: McGraw Hill. $968 \mathrm{p}$.

15. Cutler SJ, Fooks AR, Van der Poel WHM. 2010. Public Health threat of new, reemerging and neglected zoonoses in the industrialized world. Emerg Infect Dis 16:1-7. doi: 10.3201/eid1601.081467

16. Damriyasa IM, Bauer C, Edelhofer $R$, Failing $K$, Lind P, Petersen $E$, Schares G, et al. 2004. Cross-sectional survey in pig breeding farms in Hesse, Germany: seroprevalence and risk factors of infections with Toxoplasma gondii, Sarcocystis spp and Neospora caninum in sows. Vet Parasitol 126: 271286. doi: 10.1016/j.vetpar.2004.07.016

17. Dãrãbus G, Afrenie M, Olariu RT, Ilie M, Balint A, Hotea 2011. Epidemiological remarks on Toxoplasma gondii infection in Timisoara Zoo. Sci Parasitol 12: 33-37.

18. De Camps S, Dubey JP, Saville WJA. 2008. Seroepidemiology of Toxoplasma gondii in zoo animals in selected zoos in the Midwestern United States. J Parasitol 94: 648-653. doi: 10.1645/GE-1453.1

19. Dubey JP 1995. Duration of immunity to shedding of Toxoplasma gondii oocysts by cats. J Parasitol 81: 410-415. doi: $10.2307 / 3283823$

20. Dubey JP, Jones JL. 2008. Toxoplasma gondii infection in humans and animals in the United States. Int J Parasitol 38: 1257-1278. doi: 10.1016/ j.ijpara.2008.03.00

21. Dubey JP. 2010. Toxoplasmosis of animals and humans. $2^{\text {nd }}$ ed. Boca Raton, Florida: CRC Press. 313 p.
22. Epiphanio S, Sinhorini IL, CatÃoDias, JL. 2003. Pathology of toxoplasmosis in captive new world primates. J Comp Pathol 129: 196-204. doi: 10.1016/S0021-9975(03)00035-5

23. Gómez O, Chávez A, Casas E, Serrano E. 2003. Determinación de la seroprevalencia de toxoplasmosis en alpacas y llamas en la estación experimental INIA-Puno. Rev Inv Vet Perú 14 (Supp1 1): 49-53. doi: 10.15381/ rivep.v14i1.1717

24. Gorman TR, Rivoros V, Alcaino HA, Salas DR, Thiermann ER. 1986. Helminthiasis and toxoplasmosis among exotic mammals at the Santiago National Zoo. J Am Vet Med Assoc 189: 10681070.

25. Hill D, Dubey JP. 2002. Toxoplasma gondii: transmission, diagnosis and prevention. Clin Microbiol Infec 8: 634 640. 10.1046/j.1469-0691.2002.00485.x

26. Hosmer D, Lemeshow S. 2000. Applied logistic regression. New York: John Wiley. 375 p.

27. Kataranovski D, Dataranovski M, Savic I, Cakic P, Soldatovic B, Matic R. 1994. Morphometric and biochemical parameters as age indicators in the Norway rat (Rattus norvegicus Berk, 1769). Acta Vet 44: 371-378.

28. Lacave IM, Caballero TG. 2012. Atlas de inmunohistoquímica: caracterización de células, tejidos y órganos normales. Díaz de Santos. 448 p.

29. Lind P, Haugegaard J, Wingstrand A, Henriksen SA. 1997. The time course of specific antibody response by various ELISAs in pigs experimentally infected with Toxoplasma gondii. Vet Parasitol 71: 1-15.

30. Lloyd C, Stidworthy MF. 2007. Acute disseminated toxoplasmosis in a juvenile cheetah (Acinonyx jubatus). J Zoo Wildlife Med 38: 475-478. doi: 10.1638/ 2007-0016.1

31. Lopes AP, Sargo R, Rodrigues M, Cardoso L. 2011. High seroprevalence of antibodies to Toxoplasma gondii in 
wild animals from Portugal. Parasitol Res 108: 1163-1169. doi: 10.1007/s00436010-2158-6

32. Luyo C, Pinedo R, Chávez, A, Casas E. 2017. Factores asociados a la seroprevalencia de Toxoplasma gondii en cerdos de granjas tecnificadas y no tecnificadas de Lima, Perú. Rev Inv Vet Perú 28: 141-149. doi: 10.15381/ rivep.v28i1.12930

33. Milazzo C, Cagnin M, Di Bella C, Geraci F, Ribas A. 2010. Helminth fauna of commensal rodents, Mus musculus (Linnaeus, 1758) and Rattus rattus (Linnaeus, 1758) (Rodentia, Muridae) in Sicily (Italy). Rev Ibero-Latinoam Parasitol 69: 194-198.

34. Miller MA, Gardner IA, Kreuder C, Paradies DM, Worcester KR, Jessup $D A$, Dodd E, et al. 2002. Coastal freshwater runoff is a risk factor for Toxoplasma gondii infection of southern sea otters (Enhydra lutrisnereis). Int $\mathrm{J}$ Parasitol 32: 997-1006. doi: 10.1016/ S0020-7519(02)00069-3

35. Mills J, Childs J, Ksiazek T, Peters CJ, Velleca WM. 1998. Métodos para trampeo y muestreo de pequeños mamíferos para estudio virológico. Washington, USA: Organización Panamericana de la Salud. 64 p. [Internet]. Disponible en: http://www.paho.org/spanish/HCP/ HCT/hct 98104.pdf

36. Minervino AH, Soares HS, BarretoJunior RA, Neves KA, Pena HF, Ortolani EL, Dubey JP, et al. 2010. Seroprevalence of Toxoplasma gondii antibodies in captive wild mammals and birds in Brazil. J Zoo Wildlife Med 41: 572-574. doi: 10.1638/2010-0046.1

37. Mucker EM, Dubey JP, Lovallo MJ, Humphreys JG. 2006. Seroprevalence of antibodies to Toxoplasma gondii in the Pennsylvania bobcat (Lynx rufus rufus). J Wildlife Dis 42: 188-191. doi: 10.7589/0090-3558-42.1.188

38. Muñoz E, Chávez A, Casas E, Suárez F, Gavidia C, Muñoz K, Gutiérrez F. 2005. Frecuencia de anticuerpos con- tra Toxoplasma gondii en monos Cebus apella criados en cautiverio. Rev Inv Vet Peru 16: 163-168. doi: 10.15381/ rivep.v16i2.1565

39. Navarro D, Chávez A, Pinedo R, Muñoz K. 2015. Factores de riesgo asociados a la seroprevalencia de Toxoplasma gondii en mamíferos del orden Carnivora y Primates mantenidos en cautiverio. Rev Inv Vet Perú 26: 497-508. doi: 10.15381/rivep.v26i3.11175

40. Nishikawa Y, Claveria FG, Fujisaki K, Nagasawa H. 2002. Studies on serological cross-reaction of Neospora caninum with Toxoplasma gondii and Hammondia heydorni. J Vet Med Sci 64: 161-164.

41. Pavlin BI, Schloegel LM, Daszak P. 2009. Risk of importing zoonotic diseases through wildlife trades, United States. Emerg Infect Dis 15: 1721-1726. doi: 10.3201/eid1511.090419

42. Pellizzaro M1, Conrado FO, Martins CM, Joaquim SF, Ferreira F, Langoni H, Biondo AW. 2017. Serosurvey of Leptospira spp and Toxoplasma gondii in rats captured from two zoos in Southern Brazil. Rev Soc Bras Med Tro 50: 857-860. doi: 10.1590/0037-86820138-2017.

43. Pena HF, Marvulo MF, Horta MC, Silva MA, Silva JC, Siqueira DB, Lima $P A$, et al. 2011. Isolation and genetic characterization of Toxoplasma gondii from a red-handed howler monkey (Alouatta belzebul), a jaguarundi (Puma yagouaroundi), and a blackeared opossum (Didelphis aurita) from Brazil. Vet Parasitol 175: 377-381. doi: 10.1016/j.vetpar.2010.10.015

44. Pimentel JS, Gennari MS, Dubey JP, Marvulo MFV, Vasconcellos AS, Morais, ZM, Silva JCR, et al. 2009. Inquérito sorológico para toxoplasmose e leptospirose em mamíferos selvagens 
neotropicais do zoológico de Aracaju, Sergipe. Pesqui Vet Brasil 29: 1009-1014. doi: 10.1590/S0100-736X2009001200010.

45. Richomme C, Afonso E, Tolon V, Ducrot C, Halos L, Alliot A, Perret C, et al. 2010. Seroprevalence and factors associated with Toxoplasma gondii infection in wild boar (Sus scrofa) in a Mediterranean island. Epidemiol Infect 138: 1257-1266. doi: 10.1017/S0950268810000117

46. Riemann HP, Behymer DE, Fowler ME, Schulz T, Lock A, Orthoefer JG, Silverman S, et al. 1975. Prevalence of antibodies to Toxoplasma gondii in captive exotic mammals. J Am Vet Med Assoc 165: 798-800.

47. Ruiz N, Casas E, Suarez F, Díaz D, Fernández V. 2012. Frecuencia de anticuerpos contra Neospora caninum y Toxoplasma gondii en canes con signos clínicos de afección neuromuscular. Rev Inv Vet Peru 23: 441-447.

48. Scott M. 1988. The impact of infection and disease on animal populations: implications for conservation. Conservation Biology 2: 40-56. doi: 10.1111/j.15231739.1988.tb00334.x

49. Sedlák K, Bartová E. 2006. Seroprevalences of antibodies to Neospora caninum and Toxoplasma gondii in zoo animals. Vet Parasitol 136(Suppl 3-4): 223-231. doi: 10.1016/j.vetpar.2005.11.021

50. Silva JC, Gennari SM, Ragozo AM, Amajones VR, Magnabosco C, Yai LE, Ferreira-Neto JS, et al. 2002. Prevalence of Toxoplasma gondii antibodies in sera of domestic cats from Guarulhos and São Paulo, Brazil. J Parasitol 8: 419-420. doi: 10.1645/00223395(2002)088[0419:POTGAI]2.0.CO;2

51. Silva JC, Marvulo MFV, Días RA, Ferreira F, Amaku M, Adania CH, Ferreira-Neto JS. 2007. Risk factors associated with seropositivity to Toxoplasma gondii in captive neotropical felids from Brazil. Prev Vet Med 78: 286-295. doi: 10.1016/j.prevetmed.2006.10.013
52. Silva RA. 2005. Antibodies to toxoplasmosis in horses from Pantanal, Brazil. Vet Zootec 12: 20-25

53. Smith DD, Frenkel JK. 1995. Prevalence of antibodies to Toxoplasma gondii in wild mammals of Missouri and East Central Kansas biologic and ecologic considerations of transmission. J Wildlife Dis 31: 15-21. doi: 10.7589/ 0090-3558-31.1.15

54. Sobrino R, Cabezon O, Millan J, Pabon M, Arnald MC, Luco DF, et al. 2007. Seroprevalence of Toxoplasma gondii antibodies in wild carnivores from Spain. Vet Parasitol 148: 187-192. doi: 10.1016/j.vetpar.2007.06.038

55. Spencer JA, Joiner KS, Hilton CD, Dubey JP, Toivio-Kinnucan M, Minc JK. Blagburn BL. 2004. Disseminated toxoplasmosis in a captive ring-tailed lemur (Lemur catta). J Parasitol 90: 904906. doi: 10.1645 / GE-249R

56. Tidy A, Fangueiro S, Jitender P. Dubey, Cardoso L, Lopes AP. 2017. Seroepidemiology and risk assessment of Toxoplasma gondii infection in captive wild birds and mammals in two zoos in the North of Portugal. Vet Parasitol 235: 47-52. doi: 10.1016/j.vetpar.2017.01.004

57. Troncoso IE, Uribe PA, Arrué KC, Valenzuela AA, Wiethuchter C. 2015. Seroprevalencia de Toxoplasma gondii en gatos (Felis catus, Linnaeus 1758) residentes en San Carlos, Chile. Rev Med Vet 29: 23-31. doi: 10.19052/ mv.3443

58. Ullmann LS, Da Silva RC, De Moraes $W$, Cubas ZS, dos Santos LC, Hoffmann JL, Moreira N, et al. 2010. Serological survey of Toxoplasma gondii in captive Neotropical felids from Southern Brazil. Vet Parasitol 172:144146. doi: 10.1016/j.vetpar.2010.04.013

59. Vitaliano SN, Soares HS, Pena HFJ, Dubey JP; Gennari SM. 2014. Serologic evidence of Toxoplasma gondii infection in wild birds and mammals from southeast Brazil. J Zoo Wildl Med 45:197-199. doi: 10.1638/ 2013-0179R.1 
60. Weiss LM, Kim K. 2007. Toxoplasma gondii - The model apicomplexan: perspectives and methods. London, UK: Academic Press. 1085 p.

61. Wiener Lab. 2008. Prueba de hemaglutinación indirecta (HAI), para la detección de anticuerpos contra Toxoplasma gondii. Argentina [Internet]. Disponible en: http://www.wiener-lab.com.ar/wien- er/catalogo/archivos/6395_toxotest_hai_sp.pdf

62. Yai LE, Ragozo AM, Soares RM, Pena HF, Su C, Gennari SM. 2009. Geneticdiversity among capybara (Hydrochaeris hydrochaeris) isolates of Toxoplasma gondii from Brazil. Vet Parasitol 162: 332-333. doi: 10.1016/j.vetpar.2009.03.007 